\title{
The ASDEX Upgrade Digital Video Processing System for Real-Time Machine Protection
}

\author{
Reinhard Drube ${ }^{\mathrm{a}}$, Gregor Neu ${ }^{\mathrm{a}}$, Richard H Cole ${ }^{\mathrm{b}}$, Klaus Lüddecke ${ }^{\mathrm{b}}$, \\ Tilmann Lunt ${ }^{\mathrm{a}}$, Albrecht Herrmann ${ }^{\mathrm{a}}$, ASDEX Upgrade Team \\ ${ }^{a}$ Max-Planck-Institut für Plasmaphysik, EURATOM Association, Boltzmannstr. 2, 85748 Garching, Germany \\ ${ }^{b}$ Unlimited Computer Systems GmbH, Seeshaupterstr. 15, 82393 Iffeldorf, Germany
}

\begin{abstract}
This paper describes the design, implementation, and operation of the Video Real-Time diagnostic system (VRT) of the ASDEX Upgrade plasma experiment and its integration with the ASDEX Upgrade discharge control system (DCS). Hot spots produced by heating systems erroneously or accidentally hitting the vessel walls, or from objects in the vessel reaching into the plasma outer border, show up as bright areas in the videos during and after the reaction. A system to prevent damage to the machine by allowing for intervention in a running discharge of the experiment was proposed and implemented. The VRT was implemented on a multi-core real-time Linux system. Up to 16 analog video channels (color and b/w) are acquired and multiple regions of interest (ROI) are processed on each video frame. Detected critical states can be used to initiate appropriate reactions - e.g. gracefully terminate the discharge. The system has been in routine operation since 2007
\end{abstract}

Keywords: Real-Time, Image Processing, Machine Protection.

\section{Introduction}

The use of standard analog $\mathrm{b} / \mathrm{w}$ video cameras for the visible light spectrum provides a good basis for detecting different thermal phenomena in our experimental environment [1]. These phenomena include glowing of built-in components or tile edges touched by the plasma at varying locations. In fusion experiments such as ASDEX Upgrade the heating and subsequent glowing of vessel components is slow enough to be captured by standard analog video frame rates and reacted to in realtime. In figure 2 the computed brightness of observed image parts of a manipulator (movable probe) touching the plasma is shown over time. The time interval between consecutive frames is $40 \mathrm{~ms}$ (corresponding to European analog video standards). The withdrawal of the manipulator can be observed clearly. The horizontal stripes of the glowing manipulator head moving to the right result from the read-out of interlaced odd and even half frames with a time shift of $20 \mathrm{~ms}$. The shown image detail is identical for all pictures.

The principal advantages of using conventional cameras instead of infrared systems [2-4] are: a widespread variety of (even very small) form factors, allowing for easier installation in the limited space available in observation ports and moderate prices due to a wide choice of suppliers. The latter also guarantees availability of standard video components for the next years. An important requirement in this context was the re-use of already installed analog camera equipment. Unlike most digital cameras, analog cameras operate reliably in the harsh electromagnetic environment around a fusion experiment. This results in higher robustness and in more stable operation - features which are particularly desirable in protection systems. For digital processing of analog video signals frame grabbers are mandatory. Using fiber-optic video transmission avoids electromagnetic distortions of the analog signal on the way from the camera to the frame grabber.

The eventual reaction to a detected, potentially dangerous glow must be performed by the Discharge Control System (DCS) [5] which has access to all actuator systems (heating systems, fueling systems, manipulators, coils), and can initiate appropriate counteractions. This requires that a real-time communication path exists between VRT and DCS. To preserve the safety-system characteristics of the VRT, communication was designed to be minimal, essentially one-way (from VRT to DCS) with only the necessary synchronization information flowing in the other direction.

A further important requirement, driven by the continuously evolving experimental boundary conditions, was the quick and simple configurability of monitored areas (regions of interest, ROI) with arbitrary topologies, and of different recognition algorithms and their corresponding parameters between experiment runs.

In this paper we present the design concept, the hardware used, and the occurring and solved problems in achieving robust operation of the whole system.

\section{System architecture}

Given the decision to use analog cameras, a real-time operating system had to be found for which at least one manufacturer of frame grabber boards provides driver 


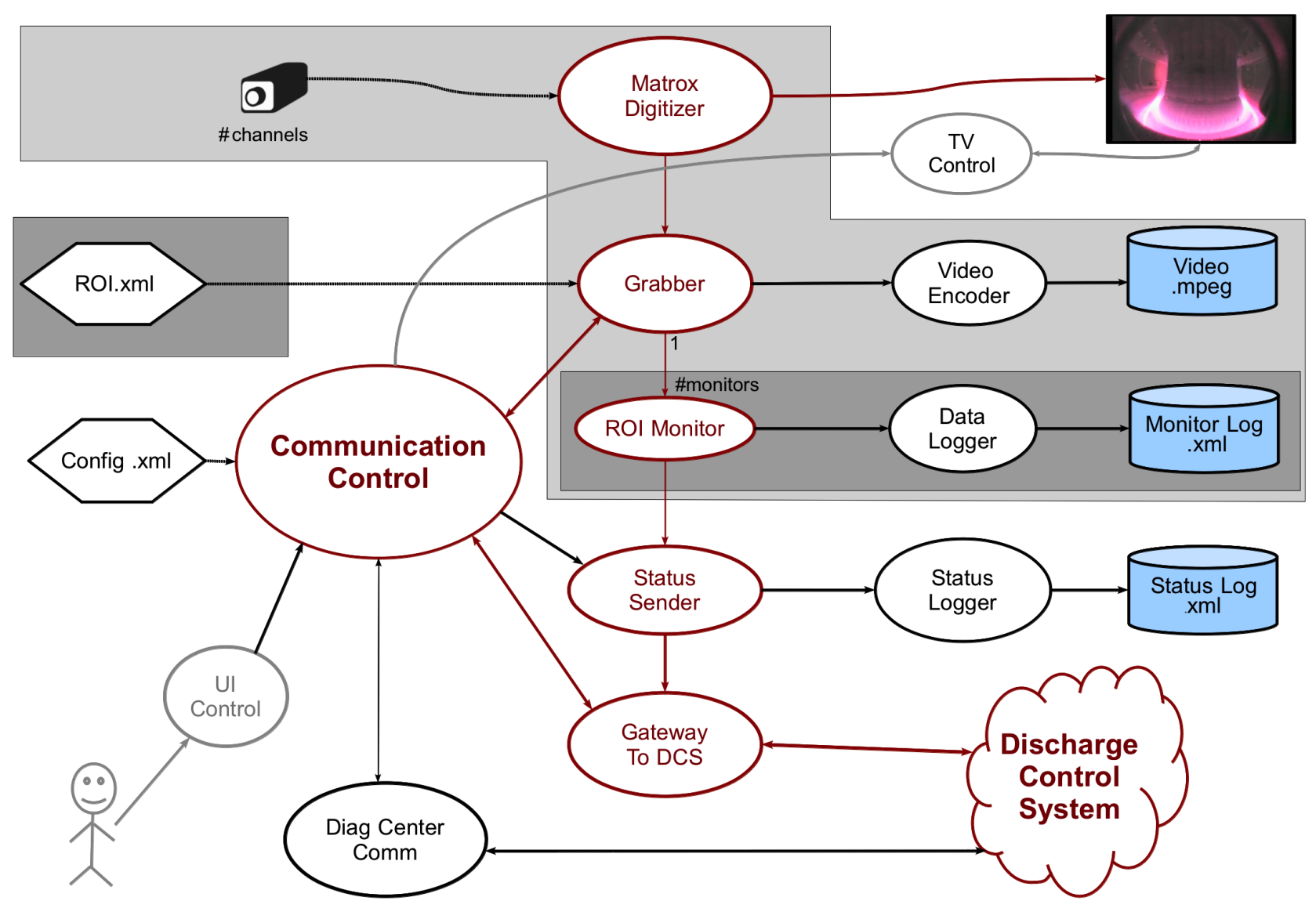

Fig.1 data flow and communication paths

software. Most manufacturers provide drivers mainly for MS Windows. The demand of serving many independent, asynchronously running video channels, however, resulted in the requirement for an operating system capable of multi-threaded real-time operation. MS Windows' perceived weakness in this aspect prompted us to look for a candidate within the Linux family. The final choice was RedHawk (64 bit for sufficient address space), a real-time modification of the Red Hat system adapted by Concurrent Computer Corporation [5]. RedHawk guarantees a user-level application response to an external event in less than 15 microseconds on most platforms. Optimizing the system response is done by shielding some of the CPU cores from non-real-time threads. Dedicated threads and interrupts can be bound to these shielded CPU cores to provide minimum response time. For this operating system, Matrox provides compatible drivers for their frame grabber boards [6].

The selected Matrox Morphis Quad frame grabber provides four independent BNC video input connectors which can be operated simultaneously at full speed to grab original video frames and requires a PCI-X slot (meanwhile other form factors are available:PCIe, PCI104). PAL, NTSC, CCIR, and RS-170 cameras are supported. The signal depth is eight bits. Grabber cards with built-in hardware MPEG encoders are not useful for our purposes. We installed four Matrox Morphis Quad frame grabber cards. The maximum possible number of video channels depends on how many of them are to be recorded in color, which consumes three times the PCI bus bandwidth compared to recording in $\mathrm{B} / \mathrm{W}$. The selected iHawk 864 system of Concurrent Computer Corporation is a quad-socket Dell PowerEdge 6850 server equipped with four Xeon microprocessors running at $2.8 \mathrm{GHz}$ with dual core and hyper-threading technology which offers 16 virtual CPUs. The board provides three PCI-X slots hosting three of the Matrox Morphis cards. The $4^{\text {th }}$ card is accommodated by an expansion box connected via PCIe to the main system.

The system has 16 Gbytes of memory and a RAID5 disc system. During an experimental plasma discharge of ASDEX Upgrade the system records about 150 Mbytes of data to be archived. The local disc system can buffer these data for several weeks. The system is designed to be capable of continuous operation, limited only by diskspace.

Two independent network controllers, provide the connections to the real-time discharge control system as well as to the non-real-time long-term archive systems. To optimize fast network response, the network interrupts are assigned to a dedicated shielded CPU.

The Matrox Imaging Library (MIL-Lite), which includes the drivers, is the mandatory interface to the frame grabber boards. This encapsulated software has a highly multi-threaded design. For every grabbed video channel up to nine threads are created. For most of them the intended function cannot be identified from outside. Matrox does not provide the source code for these drivers. The unknown internals of these threads prevent 
the possibility of shielding relevant real-time threads from the rest.

Due to the sensitivity of the Matrox frame grabber cards and of the MIL-Lite to video signals of poor quality (noise, ground loops or bad sync signals), special care had to be taken when setting up the video transmission lines. To achieve a good signal/noise ratio, the video signal transmission between camera and frame grabber is done with analog optical signal transmission. Bad shielding of the camera power supplies results in signal distortions due to harmonics of the (European) 50 $\mathrm{Hz}$ power frequency that inhibit reliable continuous frame grabbing.

To turn it into an effective machine protection system, VRT had to be interfaced to the existing discharge control system (DCS) [7]. The DCS performs all the activities necessary to set up and execute a plasma discharge. A cluster of real-time controllers runs algorithms to control plasma parameters such as density, plasma shape, etc. and to continuously monitor technical and physical parameters of the current experiment. To perform its tasks, the DCS controllers exchange signals with real-time diagnostics through a diagnostic gateway process and access a variety of actuator systems which affect plasma properties

\section{Processing concept}

The system is designed as a set of independently running, data driven processing pipelines, each pipeline serving one video stream. The real-time data flow of a pipeline is shown as a red line in fig. 1. The most important real-time processing pipeline is represented by red lines between the Matrox Digitizer (being the primary data source) and the Discharge Control System (DCS) as the last data consumer in this picture. Ovalshaped symbols represent single threads. The light gray box exists once for each video channel while the dark gray box exists once for each configured ROI monitor per video channel. If no ROI monitor is configured for a video channel the dark gray box is absent. As described later, the UI thread serves the user interface of the system. Once configured and started, the Matrox device drivers and the Matrox Imaging Library (MIL-Lite) post completed frames from the cameras via a callback routine in the VRT. Administration (creation, termination) and supervision of the VRT threads is performed by the central communication control thread.

Well engineered multi-threading increases the robustness of a system. Local failures and possible nonstandard behavior of external system components (e.g. archiving) do not result in malfunction of the entire system. Extensive self-monitoring and resynchronization to a unique initial system state - even after unexpected external communication events - must be done automatically to increase robustness. To act as a safety system, machine protection relevant threads are given higher priority by running them in real-time mode. These threads must be conceived very carefully to avoid hidden deadlocks (waiting for events that may never occur, race conditions). Explicit loop counters are used wherever possible to avoid while-until constructs. No iterative algorithms are allowed even if convergence criteria are well known. Infrastructure tasks (e.g. archiving, logging) are implemented as detached threads with lower priority. They will not disturb the real-time parts of the system if their resources are temporarily unavailable (e.g. mass storage for archiving).

The normal operation mode of the VRT is fully automatic and coordinated (phase control and configuration) with the discharge operation via the Diag Center Communication thread. Human interaction is possible through the User Interface, e.g. for testing purposes. The desired configuration for the VRT is controlled by a main Config XML file which contains all required information to describe the video channels to be processed in the next discharge and is read during the discharge preparation phase. In this phase the system is also reset to a unique, well-defined initial system state. Archiving threads from previous discharges which have not terminated, however, will continue executing. Now, for each video channel, the processing pipeline is set up by creating a Grabber thread and a corresponding Matrox Digitizer thread with real-time priority. The Matrox thread hosts the interrupt handler for incoming video frames. For each Morphis board up to four interrupt handlers are assigned to a dedicated (shielded) CPU to achieve minimal response time. MIL-Lite provides a hook for the user defined callback routine. This routine accesses two important devices: the input video device and the Time Digital Control device (TDC) which is initialized by the TDC thread at program startup. The TDC provides a high resolution systemwide unique time base [8]. The callback routine annotates each frame with a 64-bit nanosecond time stamp, channel, and frame count information.

If it is intended that the Grabber threads handle machine protection relevant parts of the images (ROIs), these are defined in separate ROI XML description files read by the Grabber threads during the preparation phase. Fully qualified names and all necessary properties of the ROI XML files such as needed detection algorithm, safety-relevance, and alarm thresholds are set in the main Config XML file. A ROI can be composed of possibly many disjoint parts of the whole image. Although MIL-Lite provides rectangular ROIs to be grabbed as sub frames, we decided to grab entire frames and to define ROIs of arbitrary shape, because crucial parts of a picture have no rectangular shape in normal cases. The advantages of grabbing entire frames are that when reviewing the recorded images one can observe events ("false negatives" cauesed by new plasma shapes or heating conditions leading to overheating of previously unaffected regions) beyond the currently defined ROIs and that the previously captured frames can easily be used to create new ROIs by simple eroding image processing techniques Different ROIs may overlap. For each ROI one or more ROI monitors can be defined in the main Config XML file. Each ROI monitor is characterized by an optional filter, a detection 
algorithm resulting in a computed brightness, alarm thresholds, and a switch for local alarm de-activation. If de-activated, the monitor operates for documentation purposes only. For each ROI monitor a separate thread is created. De-activated ROI monitor threads have lower priority than the activated ones. Additionally, up to four of the video channels can be selected to be displayed on a large TV monitor in the Control Room of ASDEX Upgrade. If a channel is marked for display, the corresponding Matrox thread copies the frame to the display source buffer. An independent Display thread wakes up periodically every $40 \mathrm{msec}$ and transfers the frame to the display via OpenGL double buffering technique.

During plasma operation the system acts as a machine protection system. The hardware interrupt created by an incoming video frame wakes up the Grabber process asynchronously via the callback routine. Each grabbed video frame receives an absolute TDC time stamp. The process enqueues the frame in the processing pipeline. Fig. 1 shows the real-time paths as red lines. Having finished processing, the Grabber thread sends the frame to the corresponding video stream encoder which produces MPEG video files on the fly for documentation and archiving purposes. Finally, the frame buffers for the processed frames are given back to the central frame buffer pool for re-usage

\section{Algorithms and Integration with Discharge Control System}

For each configured ROI monitor optionally the prerecorded full-frame offset is subtracted from each corresponding pixel. The 8-bit pixel value is renormalized to 0...255: The denominator avoids division by zero under any circumstances

$$
\text { pixel }_{\text {normalized }}=\frac{255\left(\text { pixel }_{\text {orig }}-\text { pixel }_{\text {background }}\right)}{\left(256-\text { pixel }_{\text {background }}\right)}
$$

Optionally an image filter (e.g. $3 \times 3$ Median) is

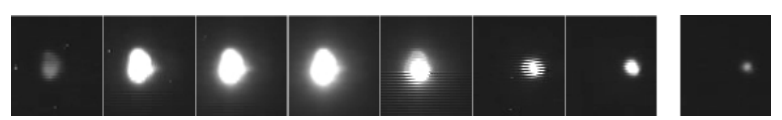

Fig.2 The glow-up of a reciprocating manipulator within a series of seven subsequent pictures (time distance $40 \mathrm{~ms}$ each). The last picture is recorded $0.5 \mathrm{sec}$ later.

applied to the frame before the selected algorithm is executed

At the moment we operate with three different algorithms:

Brightness: if it is well known where built-in components like manipulators may overheat, we define a ROI covering these components and calculate an integrated intensity over this ROI, normalized by the number of pixels (see fig. 2).

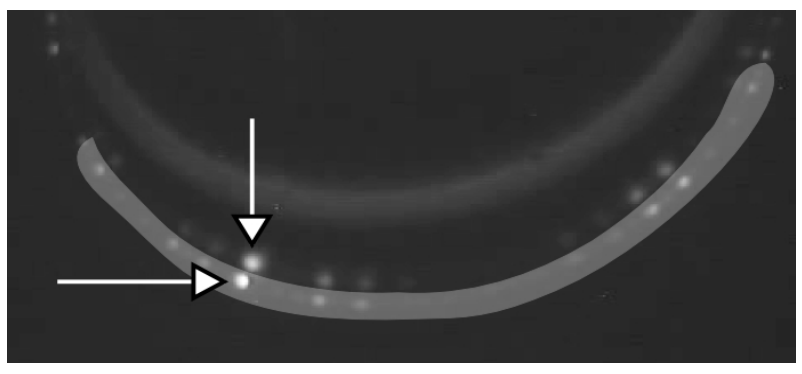

Fig.3 The horizontal arrow denotes the brightest hot spot in the gray marked ROI. The vertical arrow denotes the reflection of this hot spot which is unimportant for protection tasks.

Hot spots: If the challenge is searching for first traces of overheating somewhere in a ROI (for example tile edges in the plasma divertor) we look for the brightest $2 \times 2$ or $3 \times 3$ pixel square and use it for our calculations. Because of the time shift between odd an even half frames, moving particles will not produce intensities above 0.67 in multi-pixel squares (see fig. 4) Setting the limit to 0.99 therefore is a simple way to avoid false positives. Due to the implemented linear search algorithm this algorithm is much more time consuming and can be used for machine protection only for $25 \%$ of a frame without violating the real-time conditions (see fig. 3). If the processing time for such a ROI monitor approaches $40 \mathrm{msec}$, it is safer to use the $2 \times 2$ version which needs half of the computing time. Because we want to detect spots in the optical focus, larger or asymmetric areas need not be considered (see the hot spot at fig. 3).

Moving particles: The third algorithm looks for moving particles which are larger than the one-pixel-size neutron induced artifacts. To distinguish between moving particles and stationary glowing parts this algorithm can optionally operate with anti-correlation of

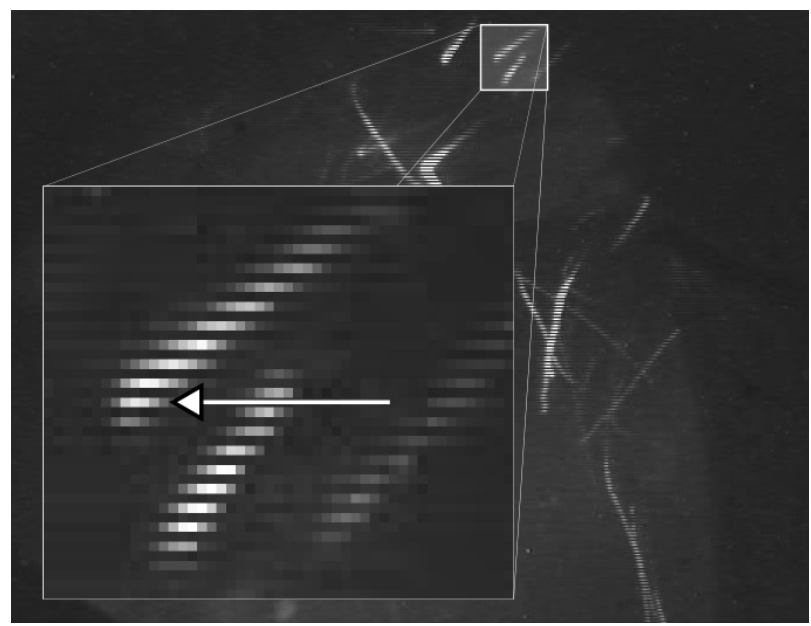

Fig.4 Example of detected moving particles. Note the streaky traces of the glowing particles which result from being recorded only in one of the two half frames. The horizontal arrow denotes one pixel line within the frame. The pixel lines above and below have a time shift of $20 \mathrm{msec}$. 
the two half frames of one captured full frame which have a system immanent time shift of $20 \mathrm{~ms}$. To avoid neutron induced artifacts a previous (3x3)-median filtering on half frames is mandatory. After median filtering we apply a linear-intensity-weighted (3x3)Laplace filtering on both half frames separately. Negative values are set to zero. Being a two-dimensional $2^{\text {nd }}$ derivative multiplied with the brightness of the original half frame, this method is sensitive for bright high spatial frequency events. Dark noise flickering has no effect even if the $2^{\text {nd }}$ derivative results in large values. This is damped by scaling with the original brightness. All these measures result in an algorithm that cannot be disturbed by $\mathrm{H}$-alpha radiation from the plasma which has much lower spatial frequency. So the risk of false positives is minimal. Moving particles must be in optical focus to be detected, otherwise the spatial frequency decreases too much to be detected (see fig. 4).

All algorithms produce numerical intensities between 0 and 1 which are then compared to pre-configured thresholds. Each ROI monitor has its own status object. Depending on the result of the calculated intensity with respect to the pre-defined thresholds, the corresponding value is set in the monitor's status object. This global status is updated after each ROI monitor processing is completed. Every 40 milliseconds the status sender collects all status objects and maps them to a global status which is sent to the Discharge Control System. Currently the global status contains exactly one bit which when set amounts to a request to execute a softstop, i.e. to terminate the running experiment. DCS, however, has the final say whether to fulfil the request: in fact, depending on the overall experiment state, (available to the DCS) it may make sense to ignore this status bit. If a camera signal fails for which we have configured a safety relevant ROI, no updated status object of this ROI monitor is written. Within the status sender there exists a counter for each status object recording how many successive frames are missing. After 3 missing frames $(120 \mathrm{msec})$ the system produces warnings. This should not happen but it can occur if a camera runs into electronic problems. After 10 missing frames $(400 \mathrm{msec})$ we assume a complete camera failure. The status sender then also requests the termination of the running experiment. This behavior is a compromise between acting safely enough and avoiding false positive terminations of the running experiment due to electronic camera distortions.

The 'run' command issued through Diag Center starts the real-time communication phase. Configuration changes are no longer possible at this stage. All configured threads are in operation. Half a second before the plasma ignites (an information provided by the DCS through the diagnostic gateway) about 10 frames per channel are grabbed, added up, averaged, and stored for the later offset compensation mentioned above. Ideally, these frames should show only black pictures. Especially older CCD cameras show a more or less gray background instead, often with dead pixels killed by neutron damage. With these reference pictures we can subtract the inherent background gray level of every recorded camera before applying the configured algorithm as described above.

DCS provides also the information when the experimental run is finished. After that the frame grabbing is stopped, recorded protocol data are sorted (time, channel), formatted, and sent to mass storage for long term documentation. Also we record statistical diagnostics of time jitter for every video channel and every ROI monitor. These data give a reliable indication on whether we can add further ROI monitors without running into performance problems. During very long discharges logging may also be initiated if the amount of protocol data exceeds some watermarks. The duration of one experimental run is limited only by the capacity of the built-in hard disc. The system itself is capable of steady state operation. The raw MPEG streams (I-frame only coded, weak compression) recorded during the experimental run do not contain data about their own length - an information which is necessary to enable scrolling in any available video player. For that purpose they must be re-encoded and are then also archived for long term access. This is done with the ffmpeg package [9]. Archived streams are also a good test basis for future algorithm development. The recorded frames can be reinserted into the detection algorithms to see what would have happened under changed algorithm conditions.

In addition to the main system described here, we have installed a smaller test system operating the same RedHawk with two Matrox Morphis Quad boards to check new software modifications. For that system a possible discharge termination request is disregarded by default. This test system also offers a form of soft redundancy. It can be reconfigured to take over the principal tasks of the main VRT system in about one hour for a maximum of 8 video channels.

\section{Summary and Outlook}

The system described is in stable standard operation for all plasma experiments at ASDEX Upgrade. Achieving the current reliability level was a lengthy process of trial and error that involved switching between several releases of the RedHawk operating system (from 5.2 up to 6.3) until we reached a technical freeze at RedHawk 5.2 which features a clear kernel structure and stable threading behavior together with MIL-Lite.

Within the last experimental campaigns $2 \%$ of the plasma discharges were terminated by VRT to avoid harmful situations like dangerous overheating. Examples are shown in fig. 2-4. The different detection algorithms fulfill their tasks reliably. False positive alarms could be reduced to less than $1 \%$ of all plasma discharges. False negatives are hard to define and therefore to count and are often caused by novel configurations of plasma and/or heating systems. Considered extensions are: providing more status information to the DCS system than just the request to terminate the running experiment, using multiple thresholds and the possibility of defining 
tailored reaction mechanisms in the DCS. More flexible answers can be supported: reciprocating probes such as manipulators could be moved to secure places, external heating systems could be switched off, or the plasma position could be shifted. The usability of digital cameras for better time resolution vs. system robustness will be tested in near future.

\section{References}

[1]: A.Herrmann et al: Fusion Eng. Des. 86 (2011) 530-534

[2]: D. Guilhem, et al: Fusion Eng. Des. 74 (2005) 879-883

[3]: Reichle et al: J. Nucl. Mater. 390-391 (2009) 1081-1085

[4]: Reichle et al: Rev. Sci Instrum 81 10E135 (2010)

[5]: http://www.ccur.com/concurrent_redhawk_linux.aspx

[6]: http://www.matrox.com/imaging/en/products/software/mil/

[7]: Raupp et al: Fusion Eng. Des. 74 (2005) 697-705

[8]: Raupp et al: Fusion Eng. Des. 81 (2006) 1747-1751

[9]: http://www.ffmpeg.org/ 